\title{
Extracting PD therapy from coffee
}

\section{By Michael J. Haas, Senior Writer}

A U.S. team that included researchers from Signum Biosciences Inc. has identified a small molecule in coffee that increased motor function in a mouse model of Parkinson's disease. ${ }^{1}$ By blocking disease progression, the compound may be superior to marketed PD drugs that only improve symptoms.

The Signum-led group now will use a $\$ 295,000$ grant from The Michael J. Fox Foundation for Parkinson's Research to develop the compound-and others acting via the same mechanism-to treat PD.

Extensive phosphorylation of the $\alpha$-synuclein (SNCA) protein at serine 129 is thought to be a driver of PD progression. Toxic aggregates

"Going after the protein's
aggregation to get a
disease-modifying effect
is the right strategy in
Parkinson's disease."
-Ari Azhir, Neuraltus
Pharmaceuticals Inc.

"Going after the protein's aggregation to get a disease-modifying effect is the right strategy in Pharmaceuticals Inc.

products because, according to team leader M. Maral Mouradian, many plant-derived compounds have beneficial effects on cognition and neuronal function.

In particular, studies have linked coffee consumption to reduced risk of PD and Alzheimer's disease (AD)., ${ }^{2,3}$

Indeed, the screen identified eicosanoyl-5-hydroxytryptamide (EHT) - a compound found in coffee-as a low micromolar inhibitor of PP2A demethylation.

In mouse models of PD that expressed human SNCA, EHT decreased SNCA phosphorylation and aggregation in neurons, lowered neurodegeneration and improved the animals' performance in sensorimotor tests compared with vehicle (see Figure 1, "Keeping PP2A methylated to treat Parkinson's disease").

"Compounds such as EHT have the potential to modulate the causes of neurodegeneration in Parkinson's disease-and therefore slow down or halt its progression-rather than simply providing temporary symptom relief" as current approved therapies do, Mouradian told SciBX.

Mouradian is a professor of neurology and director of the Center for Neurodegenerative and Neuroimmunologic Diseases at the Robert Wood Johnson Medical School at the University of Medicine and Dentistry of New Jersey. She has been collaborating with Signum since 2008.

of phosphorylated SNCA called Lewy bodies cause dysfunction and death of dopaminergic neurons in the brain, leading to the hallmark symptoms of the disease.

Instead of inhibiting the kinases that phosphorylate SNCA, the Signum team took an alternative approach to lowering phosphorylated SNCA levels. Their solution was to promote the activity of the brain's phosphatase enzymes, which remove phosphate groups from SNCA and other proteins.

Unlike kinases, which add phosphate groups to proteins, phosphatases have generally been considered poor drug targets, in part because it is more challenging to decrease levels of a phosphorylated protein by increasing phosphatase activity than by inhibiting kinase activity.

The team initially focused on the trimeric enzyme protein phosphatase 2 (PPP2CA; PP2A) because it accounts for more than $50 \%$ of serine/threonine phosphatase activity throughout the brain and thus seemed the most likely serine phosphatase to act on SNCA. The team therefore hypothesized that increasing PP2A activity in the brain would be beneficial in PD.

Using in vitro assays with human SNCA and an SNCA-expressing dopaminergic neuronal cell line, the team identified a specific PP2A trimer as the primary phosphatase targeting SNCA. More importantly, the group also found that a methylated isoform of the trimer was much more efficient at dephosphorylating SNCA than the nonmethylated isoform.

The team next set up an in vitro screen to help identify inhibitors of PP2A demethylation. The researchers opted to screen a library of natural
The team also included researchers from Princeton University and the University of California, San Diego School of Medicine. Data were reported in The Journal of Neuroscience.

"This paper is a major contribution in terms of understanding Parkinson's disease progression," said Todd Sherer, CEO of MJFF. Although there is no consensus among PD researchers about whether phosphorylation and aggregation of SNCA drives disease pathogenesis or is a by-product of it, this paper "is novel in that it shows that targeting phosphorylated SNCA affects disease progression and motor function."

\section{Antiprogression progress}

Signum has received a pair of 1 -year grants worth a total of $\$ 295,000$ from MJFF to develop the team's findings. The grants were awarded as part of MJFF's two funding initiatives started in 2010 to identify new therapies to treat PD. "The science reported in this paper won that competition," Sherer said.

Under the two initiatives-Alpha-Synuclein Therapeutics 2010 and Novel Hypotheses in Parkinson's Disease 2010 — three other companies and six different academic research teams were also awarded grants, added MJFF spokesperson Miranda Lanzillotti.

Given the collective evidence pointing to SNCA's involvement in PD, "going after the protein's aggregation to get a disease-modifying effect is the right strategy in Parkinson's disease," said Ari Azhir, COO and cofounder of Neuraltus Pharmaceuticals Inc.

Dinah Sah, VP of research, CNS and oncology at Alnylam Pharmaceuticals Inc., agreed that promoting PP2A's activity could be a feasible strategy to treat PD. But she noted that the paper's validation 
of PP2A as a PD target was predicated on the assumption that EHT is actually a specific inhibitor of PP2A demethylation.

Thus Sah wanted to see additional experiments demonstrating EHT's specificity for PP2A and whether there are side effects of increasing PP2A activity in neurons and other cell types. "PP2A is a ubiquitous and key enzyme that plays a role in almost all aspects of cell regulation," she said. "So changing its activity is likely to have multiple effects beyond reduction of $\alpha$-synuclein phosphorylation at serine 129."

Mouradian countered that inhibiting PP2A demethylation should be safe because the enzyme is already highly methylated in healthy cells. "Only in stressed disease states such as PD do there appear to be decreases in PP2A methylation," she said. "It seems likely that EHT will only have effects on such stressed cells."

She also said the methylated PP2A isoform that targets SNCA has only three other known targets: microtubule-associated protein- $\tau$ (MAPT; TAU; FTDP-17), $\beta$-catenin (CTNNB1) and v-raf-1 murine leukemia (RAF). "We have seen no adverse effects in animals from enhancing the activity of this pool of PP2A substrates with EHT," said Mouradian.

But even if inhibiting PP2A demethylation proves to be safe, it will be important to determine how early in the disease process the team's therapeutic approach is effective, Azhir said. "To affect disease progression, you need to target a pathway that plays an early role in that progression. If their approach can affect the earliest stages of the disease, that would be really good." Thus she wants to see experiments that explore how early in PD pathogenesis the methylation status of PP2A becomes relevant.

Sah also pointed out that-compared with therapies targeting PD symptoms-therapies targeting PD pathogenesis require longer clinical trials and thus would benefit from markers that provide early readouts of whether the therapy is working. Although a few such early markers are already available, "having additional markers would be tremendously valuable and facilitate clinical trials" of therapies that modify disease progression, she said.

Both Sah and Azhir said more work is needed to determine whether PP2A is such a marker.

With a grant from MJFF, Alnylam has small interfering RNAs targeting SNCA in preclinical testing to treat PD.

Also with a grant from MJFF, Neuraltus has NP003, a small molecule that inhibits lipid-mediated aggregation of SNCA, in preclinical testing to treat PD and Gaucher's disease. Neuraltus also has NP002, a small molecule nicotinic receptor agonist, in Phase II testing to treat levadopa-induced dyskinesia in PD patients.

\section{Come to PP2A}

Going forward, Sherer said MJFF's two grants to Signum will fund development of inhibitors of PP2A demethylation to treat PD and its investigations of PP2A methylation status as a marker of disease progression in $\mathrm{PD}$.

"We are also providing our research expertise to the company for its experiments," he said. Although the current grants are for one year, "we would renew funding and work with them as long as they continue to be successful."

Mouradian said the team's follow-on studies have shown that inhibiting PP2A demethylation with EHT is effective in multiple

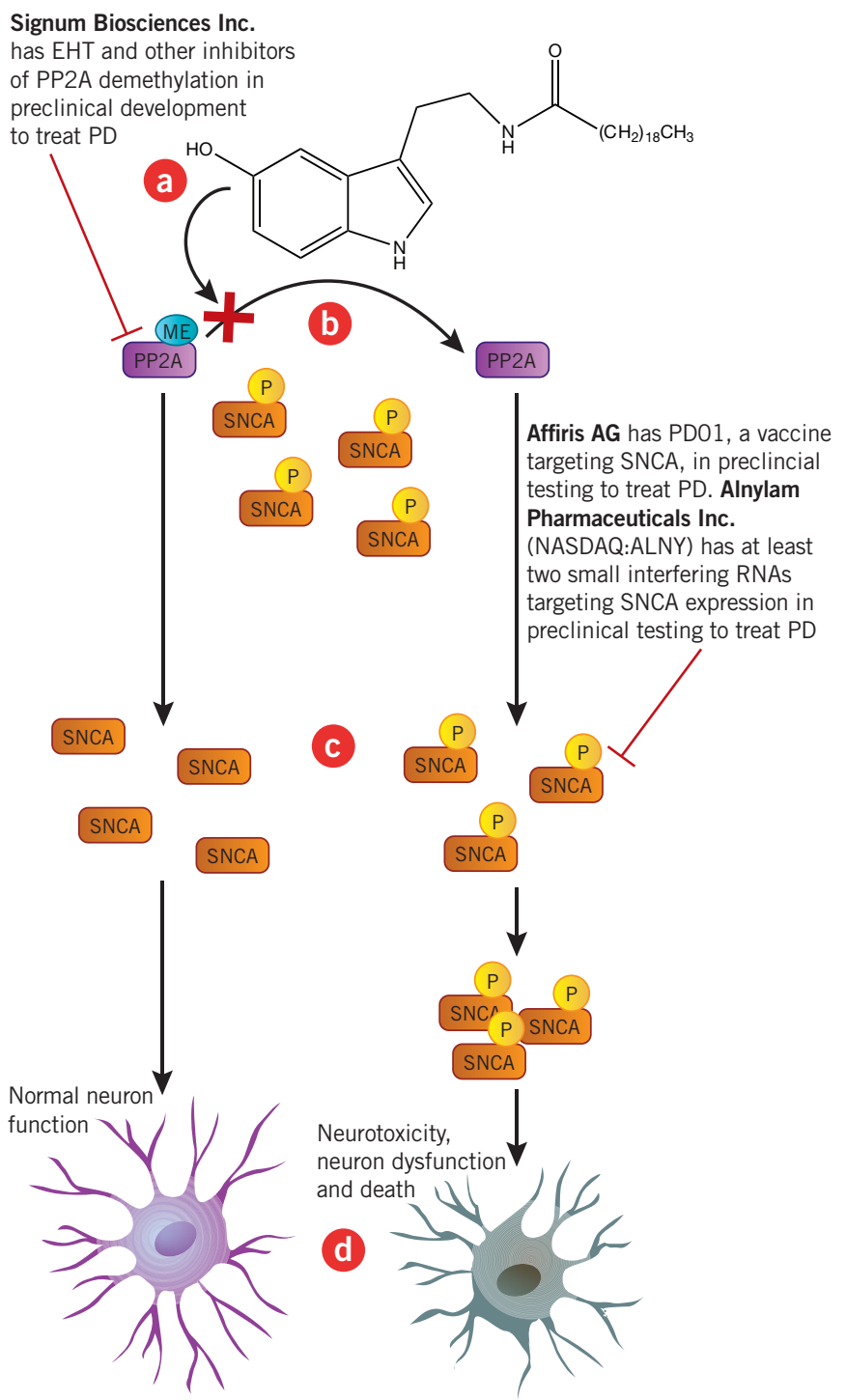

Figure 1. Keeping PP2A methylated to treat Parkinson's disease. The coffee-derived compound [a] eicosanoyl-5-hydroxytryptamide $(E H T)$ inhibits demethylation [b] of a methylated isoform of protein phosphatase 2 (PPPC2A; PP2A). Methylated PP2A leads to greater dephosphorylation [c] of phosphorylated $\alpha$-synuclein (SNCA) than nonmethylated PP2A. In turn, increased dephosphorylation of SNCA decreases aggregation of phosphorylated SNCA that causes neurotoxicity and neural cell dysfunction/death [d] in Parkinson's disease (PD).

models of PD and AD—another neurodegenerative disease in which TAU plays a role.

She added that Signum also is identifying other inhibitors of PP2A demethylation with the goal of developing first-in-class therapeutics to treat $\mathrm{PD}$ and $\mathrm{AD}$.

According to Mouradian, Signum holds patents covering the identification of inhibitors of PP2A demethylation and the use of 


\section{ANALYSIS}

those inhibitors to treat neurological diseases. The IP is available for licensing or partnering, she said.

Haas, M.J. SciBX 4(21); doi:10.1038/scibx.2011.590

Published online May 26, 2011

REFERENCES

1. Lee, K.-W. et al. J. Neurosci.; published online May 11, 2011; doi:10.1523/JNEUROSCI.6513-10.2011

Contact: M. Maral Mouradian, Robert Wood Johnson Medical School, University of Medicine and Dentistry of New Jersey, Piscataway, N.J. e-mail: Mouradian@umdnj.edu

2. Barranco Quintana, J.L. et al. Neurol. Res. 29, 91-95 (2007)

\section{TARGETS \& MECHANISMS}

3. Sääksjärvi, K. et al. Eur. J. Clin. Nutr. 62, 908-915 (2008)

COMPANIES AND INSTITUTIONS MENTIONED

Alnylam Pharmaceuticals Inc. (NASDAQ:ALNY), Cambridge, Mass. The Michael J. Fox Foundation for Parkinson's Research, New York, N.Y.

Neuraltus Pharmaceuticals Inc., Menlo Park, Calif.

Princeton University, Princeton, N.J.

Robert Wood Johnson Medical School at the University of Medicine and Dentistry of New Jersey, Piscataway, N.J. Signum Biosciences Inc., Monmouth Junction, N.J.

University of California, San Diego School of Medicine, La Jolla, Calif. 